\title{
VIBRATIONAL SPECTRUM CHARACTERIZATION OF OUTER SURFACE OF Helicobacter pylori BIOFILMS BY FUNCTIONALLY-ENHANCED DERIVATIVE SPECTROSCOPY (FEDS)
}

\author{
SIXTA L. PALENCIA ${ }^{a, b}$, APOLINARIA GARCÍA ${ }^{c}$ AND MANUEL PALENCIA ${ }^{c^{*}}$ \\ ${ }^{a}$ Laboratory of Bacterial Pathogenicity, Department of Microbiology, Faculty of Biological Sciences, Universidad de Concepción, Concepción - Chile. \\ ${ }^{b}$ Mindtech Research Group (Mindtech-RG), Mindtech s.a.s., Cali - Colombia. \\ ${ }^{c}$ Research Group in Science with Technological Applications (GI-CAT), Department of Chemistry, Faculty of Natural and Exact Sciences, Universidad del Valle, \\ Cali-Colombia.
}

\begin{abstract}
Mid-infrared spectroscopy in conjunction with Functionally-Enhanced Derivative Spectroscopy (IR+FEDS) is a powerful analytical tool for the improvement of analysis of microorganism IR spectra. The objective of this research is to characterize the outer surface of two Helicobacter pylori strains by IR+FEDS. This work is a key stage for the study of cell-cell and cell-surface interactions between microorganisms, as well as, for polymicrobial biofilm characterizations where $H$. pylori species are involved. Artificial bacterial biofilms were deposited on ultrafiltration cellulose membranes covalently modified by insertion of one spectral marker and used as sensing surface for analysis of bacterial biolayers. Biolayers were analyzed using an infrared spectrophotometer with ATR. Data were analyzed by classic procedures and by deconvolution based on FEDS transform. It is concluded that, for correct application of technique is required a minimum amount of noise in the working spectra which can be achieved by simple smoothing algorithms; in addition, reproducibility must be warranted by the implementation of standardized protocols and the use of an appropriate number of samples. It is concluded that in addition to typical signals associated with the IR spectrum of microorganisms, by FEDS, a better and more detailed description of the outer membrane of $\mathrm{H}$. pylori biofilms can be performed. In particular, it is concluded that the detecting and monitoring of cysteine-rich proteins can be satisfactorily performed by IR+FEDS.
\end{abstract}

Keywords: Helicobacter pylori; infrared spectrum; biofilm; FEDS transform; surface sensing.

\section{INTRODUCTION}

Helicobacter pylori is a microaerophilic Gram-negative bacterium; H. pylori is considered an etiologic agent since it is able to colonize the gastric mucosa in a variety of gastroduodenal diseases and peptic ulcers [1]; in addition, its study is particularly important because it is a biological risk factor for the development of gastric cancer [1-7]. It has been reported that about half the adult population in all the world is infected with this microorganism [6-7].

The surface of $H$. pylori, in addition to usual biological function that it fulfills in every microorganism, provides a vital interface for pathogen-host interactions and interaction with other microorganisms [4, 5-11]. Surface proteins of $H$. pylori, including the outer membrane proteins, can be experimentally identified by selective solubilization and sucrose gradient centrifugation for enrichment of outer membrane fractions [3, 12-13]. In H. pylori, enriched outer membrane fractions are produced by these techniques, and consequently, many surface proteins have been identified and described. However, these preparations are severely contaminated by inner membrane components and not necessarily correspond to real surface composition of the bacterium [3,13]. But also, it is well-known that different levels of molecular organization are required for the correct functioning of proteins, in consequence, destructive methods of cellular integrity produce the loss of information on the real state of surface.

On the other hand, microbial surface can be studied by several methods including Scanning Electron Microscopy (SEM), Transmission Electron Microscopy (TEM), Fluorescence Microscopy (FM), Infrared Spectroscopy (IR spectroscopy), Raman Spectroscopy, among others [14, 15]. The main limitations in the case of SEM and TEM is the high cost of equipment, the relatively high complexity of analysis, the high perturbation of samples and the obtaining of limited molecular information. On the other hand, the FM is a more economic and simpler alternative, but it requires a high perturbation of the samples to produce the fluorescence which is not inherent to many microorganisms [16-17]. IR and Raman spectroscopies are based on the use of IR radiation to produce changes of vibrational states at molecular level. In particular, IR spectroscopy is a non-destructive, fast, easy to use and highly sensitive method [18-20]. However, for microbiological analysis, the main limitations of IR spectroscopy are the high spectral similitude between spectra of different microorganisms, and the overlap and widening of adjacent signals [1820]. For the resolving of these problems usually the Fourier self-deconvolution is used [21-23]; however, its application is limited by the complexity of computation, the appearing of negative intensities from calculations, the highly sensitive to the noise, and the appearing of 'false' signals resulting of mathematical arguments without physical meaning [21]. In contrast, Functionally-Enhanced Derivative Spectroscopy (FEDS) is a simple method to obtain the spectral deconvolution and increase of spectral resolution of signals [24]. FEDS is based on the transformation of spectrum by the first-order derivative calculated from inverse of data constituting the IR spectrum, in combination with a set of simple working functions. In addition, it has the advantage that its interpretation is completely coherent with IR analysis. A complete description of principles has been previously published [24].

Given the importance of knowing the surface composition of the outer membrane in real conditions of growth, in situ operating techniques under controlled conditions producing a minimal perturbation of the biological system are highly desired. In this way, here it is proposed the study of bacterial surface by the building of artificial biolayers, or biofilms, followed by the subsequent direct analysis using spectroscopic techniques, in specific, mid-IR infrared spectroscopy (IR spectroscopy) by attenuated total reflectance (ATR) in conjunction with FEDS. Thus, the objective of this work was to characterize the outer surface of two H. pylori strains by IR+FEDS. At the present, there are no previous publications about $H$. pylori outer membrane characterization, by this technique, and with the high degree of resolution of signals achieved.

\section{EXPERIMENTAL SECTION}

\subsection{Bacterial strains and growing conditions of microorganisms}

As bacterial models were used two H. pylori strains: H. pylori ATCC J99 (American Type Culture Collection, USA) and H. pylori ATCC 43504 (American Type Culture Collection, USA). H. pylori strains were grown by inoculating in Columbia agar plates (Oxoid, England) supplemented with $7 \%$ horse blood and incubated under microaerobic conditions in the presence of $5 \%$ $\mathrm{O}_{2}, 10 \% \mathrm{CO}_{2}$ and $85 \% \mathrm{~N}_{2}$ at $37^{\circ} \mathrm{C}$ for 4 days. Bacterial growth was verified by Gram stain and urease test and SEM (Phenom PRO X, ThermoFisher Scientific). Due to high vacuum, low conductivity and regular outgassing required during SEM analysis, a gold coating was performed. 


\subsection{Preparation of cellulose sensing surface}

Ultrafiltration cellulose membranes modified using a spectral marker based on methylene diphenyl isocyanate (MDI, Aldrich, USA) were used as sensing surfaces. MDI is considered a spectral marker producing a characteristic IR signal at $\sim 2268 \mathrm{~cm}^{-1}$; this signal is absent in the IR spectra of bacteria and, in consequence, it can be used for the monitoring of background signals associated with cellulose support, and the analysis of biolayer thickness. Sensing surfaces were prepared by immersion of membranes $\left(1 \mathrm{~cm}^{2}\right)$ in dissolution of MDI (MDI/dioxane 1:1 v/v ratio) per 5 days. Catalysts were not used in order to avoid the introduction of residual signals into experimental spectra. Cellulose sensing surfaces were characterized by SEM to visualize the surface porous morphology and by Atomic Force Microscopy coupled to Raman Spectroscopy (AFM-Raman analysis) for the determination of the presence of $\mathrm{C}=\mathrm{O}$ groups associated to isocyanate units. The spectra were acquired using a high-resolution confocal LabRamHR Evolution Horiba Jobin Yvon microscope, with a $633 \mathrm{~nm}$ Edge laser line excitation source with a power of $13.3 \mathrm{~mW}$. The laser spot was focused on the sample using an optic Objective Olympus 100x VIS and NUV camera (B/S UV 50/50+Lens F125 D25).

\subsection{Preparation of samples and recording of spectra}

In order to eliminate the residual composition of growth medium on the surface, after forming the biolayer on the support, samples were successively washed with deionized water and dried using a laminar flow oven with temperature control at $40{ }^{\circ} \mathrm{C}$ per 12 hours. To warrant this stage, spectra of support and blank experiments, i.e., growth medium without bacterium, were compared with the respective spectra of biofilm samples. Finally, biolayers were analyzed using an infrared spectrophotometer with ATR using a ZnSe crystal (ATR, IR-Affinity, Shimadzu Co). Biolayer were performed in triplicate, and average spectra for each replicate were recorded by 20 scans from 500 to 4000 $\mathrm{cm}^{-1}$. Spectra are expected to show a high signal-noise ratio $\left(30 \times 10^{3}: 1\right.$ with 1-minute accumulation) and a maximum resolution of $0.5 \mathrm{~cm}^{-1}$. In addition, Michelson interferometer $\left(30^{\circ}\right.$ incident angle) equipped with Dynamic Alignment System Sealed interferometer with Automatic Dehumidifier and DLATGS detector equipped with temperature control mechanism were used. Data were extracted in file format .txt in order to carry out the analysis using a spreadsheet.

\subsection{Calculations}

Experimental IR spectrum is a set of data defined by two columns, where the first one contains the wavenumbers whereas the second one contains the respective absorbances. Thus, each row has a position in the wavenumber scale defined according to the target spectrum region. The number of points, in the working spectrum region, is defined by experimental parameters and by instrumental characteristics. Since FEDS is sensitive to residual noise in the spectrum, it is necessary to carry out a smoothing of data previous to application of FEDS transform. Details are given below.

\subsection{Pre-treatment of data}

For each region of spectrum being analyzed, data are auto-scaled with respect to the values of minimum and maximum absorbances $\left(a_{\min }\right.$ and $a_{\max }$, respectively).

$$
b_{j}=\frac{a_{j}-a_{\min }}{a_{\max }-a_{\min }}
$$

where $a_{j}$ and $b_{j}$ are the experimental and auto-scaled absorbances in the $j$-th wavenumber position. In order to avoid calculation mistakes resulting to scaling from 0 to 1 during the application of FEDS algorithm, the zero absorbance was approximated by the calculation of average value between two adjacent values of absorbance satisfying that $b_{j-1}<b_{j}<b_{j+1}$ with $b_{j}=0$ (deterministic approximation). Note that, other approximations can be performed depending of the analyst; in addition, this approximation is seen to not affect significantly the spectrum due to that it is the minimum value of absorbance detected.

\subsection{Smoothing of spectrum}

Since derivative spectrum is strongly sensitive to the noise in the original signal, the smoothing of spectral noise was decreased by the use of average-based spectral filter (ABSF) [24, 25]. ABSF is given by.

$$
\operatorname{ABSF}\left(b_{i} ; N=20\right)=\left.\frac{1}{3} \sum_{j}^{j+2}\left(b_{j}\right)\right|_{N=1} ^{N=20}
$$

ABSF is the moving average with a data window of 3 and 20 cycles $(N=20)$. For the above, for each $b_{j}, b_{j+1}$ and $b_{j+2}$ is calculated the corresponding average value, and subsequently, this procedure is repeated $N$ times. A moving average filter or $A B S F$ is commonly used with time series data to smooth out short-term fluctuations when is applied on consecutive points, therefore, $A B S F$ was used as smoothing function because the simplest algorithm. However, in order to mitigate distortion and improve noise removal, a filter of smaller width and more than one convolution were used $(j=1,2$ and $3 ; N=20)$. A comparison of smoothing methods has been recently published by Kowalski and Smyk (2018) [25].

As the spectrum line function in the absorbance domain is modified by the use of Equation 2, the same transformation of data is performed on values of wavenumber $(v)$ in order to correct the displacements respect to original spectrum (i.e., maximum points in original spectrum should be the same in the original and smoothed spectra) $[24,26]$. Therefore, for $v$ ABSF analogously is given by.

$$
\operatorname{ABSF}\left(v_{i} ; N=20\right)=\left.\frac{1}{3} \sum_{j}^{j+2}\left(v_{j}\right)\right|_{N=1} ^{N=20}
$$

Note that, depending of $N$ value used in the equations 1 and 2 a loss of data is produced, therefore, it is recommended consider this effect during the definition of working range.

\section{Theoretical Background}

For the application of FEDS transform to the mid-IR spectrum, the spectrum is considered to be a function $\mathbf{z}$ with $\mathbf{z}:\left(v_{j}, b_{j}\right)$. By FEDS, a new spectrum with sharper signals is obtained, but also, since the intensities of signals with a higher SNR are increased, the deconvolution of overlapped signals is produced.

For the obtaining of FEDS intensities, the first step is the calculation of the first-order derivative of the inverse of normalized spectrum, therefore, FEDS transform is a method of derivative spectroscopy applied on the inverse function of IR spectrum. This first stage can be easily calculated by:

$$
\frac{d \mathbf{z}}{d v}=\frac{\frac{1}{b_{j}}-\frac{1}{b_{j-1}}}{v_{j}-v_{j-1}}
$$

Assuming that $v_{j}-v_{i-1}$ is always a constant (this assumption is valid for almost all instrumental equipment), Equation 4 is rewritten to be

$$
p_{j}=\frac{1}{b_{j}}-\frac{1}{b_{j-1}}
$$

where $p$ denotes an auxiliary function formed by all values of $p_{j}$ obtained by the equation 5 . Therefore, $p$ is used in order to simplify the notation (the notation $p$ comes from the Spanish word 'primera' alluding to the use of the first derivative). Since function $p$ defines positive and negative values, the mathematical operator $\phi=1 /(|x|)^{0.5}$ is applied, where $x$ denotes any mathematical arguments, and $\phi$ is the 'key point' of data transformation. This operator is equal to scale factor defined under the wavelet concept which is used to define the general expression of 'mother wavelet' [24, 26]. Thus, for each $b_{j}$, a new function called Function $P$ transforms the values of function $p$ by

$$
P_{j}=\frac{1+b_{j}}{\sqrt{\left|p_{j}\right|}}=\phi\left(1+b_{j}\right)
$$

where $\left(1+b_{j}\right)$ is an amplification factor for the assignation of a weight congruent with experimental absorbance intensities and $P$ defines a new magnitude usually called FEDS intensity. It is important to note that $\mathrm{P}$ is not directly related with the absorbances, and therefore it is not directly related with the concentration; however, by auxiliary-fitting functions is possible to produce the re-scaling of FEDS spectrum in order to obtain a new spectrum where the main FEDS signals are equals to those obtained in the IR spectrum. 
One of the main disadvantages of FEDS is that it is a recently developed technique and therefore, unlike other methods that have data libraries, the allocation of overlapped signals is severely difficult. In general, two types of signals are obtained through FEDS: (i) signals directly related to the spectrum, which can be assigned by interpreting the infrared spectrum (signals type I) and (ii) 'pure' FEDS signals which are the result of the deconvolution process (signals type II). These signals have their origin in 'hidden' information in the line function of the spectrum, being strongly by the presence of noise on the spectrum line function. However, signals type II must be validated or contrasted with spectral and structural information in order to avoid errors. Therefore, in this work, the theoretical modeling of the infrared spectrum is used as the main tool for assigning and interpreting type II signals.

At the present, FEDS transform only has been used for quantification of water contents in mixture with organic acids [24], determination by spectral analysis the dimerization constant of acetic acid [24], nanoparticle ultraviolet spectra [27], infrared analyses of humic acids from soil samples [28], synthetic polymers [2932], biopolymers (collagen from fish scales) [33] and monitoring of binary systems (triethylamine/acetone) [34]. At the present, biofilm characterization using IR spectroscopy and FEDS have been performed only for Candida albicans [35].

\section{RESULT AND DISCUSSION}

\subsection{Characterization of cellulose sensing surface}

In the Figure 1A the AFM-Raman spectra of cellulose membrane and cellulose sensing surface are shown where it can be identified the $C=O$ signals at $1600 \mathrm{~cm}$ ${ }^{1}$, this signal is explained by the insertion of molecules of MDI on the surface of cellulose. A representation of the chemical nature of the surface is shown in Figure 1B. Thus, the surface of modified cellulose is basically formed by isocyanate groups anchored to the cellulose surface by urethane bonds. As it is expected, the membrane surface is not affected and cellulose sensing surface corresponds with some porosity degree (Figure 1C).

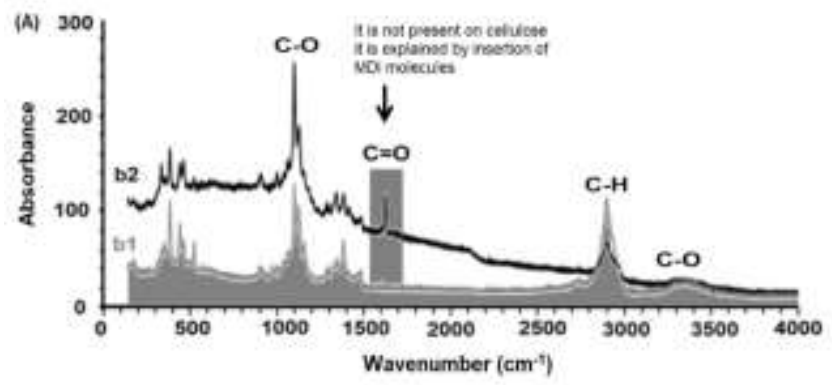

(B)

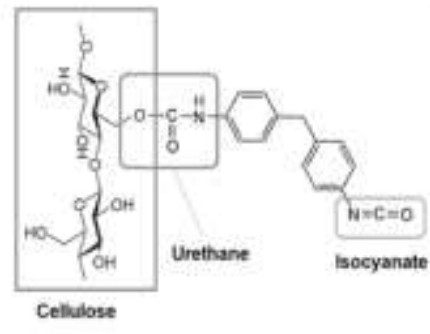

(c)

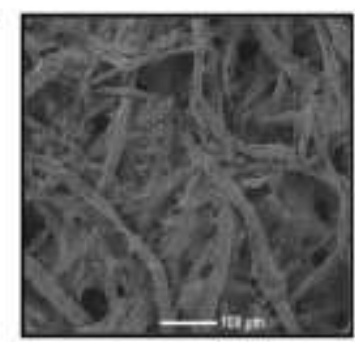

Figure 1. (A) AFM-Raman spectra of cellulose membrane and cellulose-base sensing surface (b1 and b2, respectively), (B) illustration of chemical structure of cellulose sensing surface and (C) SEM image of surface of cellulose-base sensing surface.

\subsection{Verification of $\boldsymbol{H}$. pylori biolayers}

The verification of $H$. pylori strains was verified by Gram staining (see Figure $2 \mathrm{~A}$ ) and the forming of biolayer by SEM (Figure 2B). It can be seen that bacterial cells have a bacillar form instead of coccoid shape. In consequence, it was verified that the pathogenic morphology of bacterium was analyzed in the next experiments. The above is relevant due to molecular composition of coccoid and bacillar states is not the same, and in consequence, the coccoid shape is not useful for the aim proposed.
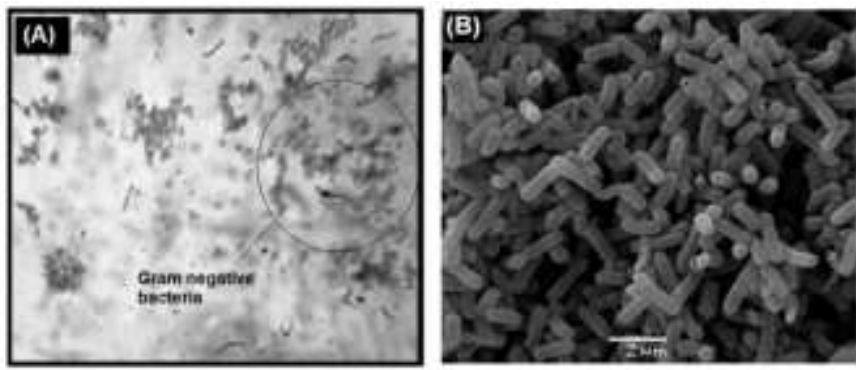

Figure 2. (A) photo of Gram staining of H. pylori strains obtained by direct captured using a confocal microscopy and (B) SEM image of $H$. pylori on the cellulose sensing surface in bacillar form.

\subsection{Reproducibility of IR spectra and effect of the noise}

During FEDS analysis the first step is the verification of quality of spectra in terms of reproducibility followed by elimination of end spectrum noise. Since there are no previous experiments of FEDS transform which can be used as reference parameters, the reproducibility analysis of spectra is a very important stage. In this way, in the Figures $3 \mathrm{~A}$ and $3 \mathrm{~B}$ are shown the IR spectra for the target strains of $H$. pylori. It can be observed that spectra are very similar for both $\mathrm{H}$. pylori strains. In addition, regions with relatively high residual noise are evidenced (see Figure $3 \mathrm{C}$ ). As an example, a comparison of FEDS transformation applied to mean working spectra with and without smoothing, for H. pylori J99, is shown in Figure 4. It can be concluded that, when FEDS transform is applied without the use of smoothing tool ABSF the results are not satisfactory due to many signals being observed; by contrast, when ABSF with $N=20$ was performed, the main signals remaining in the spectrum in a manner consistent with the IR spectrum.

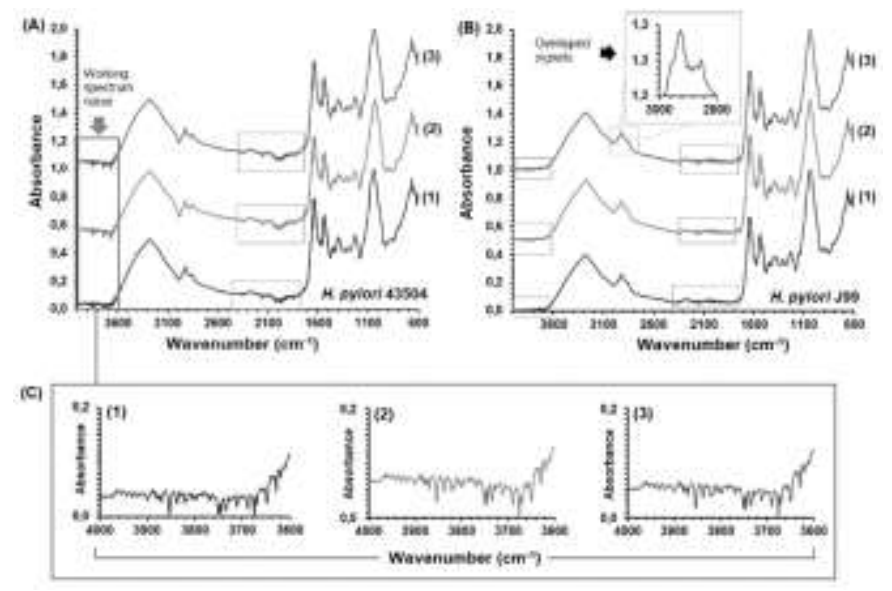

Figure 3. Mid-IR spectra of H. pylori strains: 43504 (A) and J99 (B) and, (C) Illustration of end spectrum noise for region between 4000 and $3600 \mathrm{~cm}^{-1}(1,2$ and 3 indicates the respective replicates).

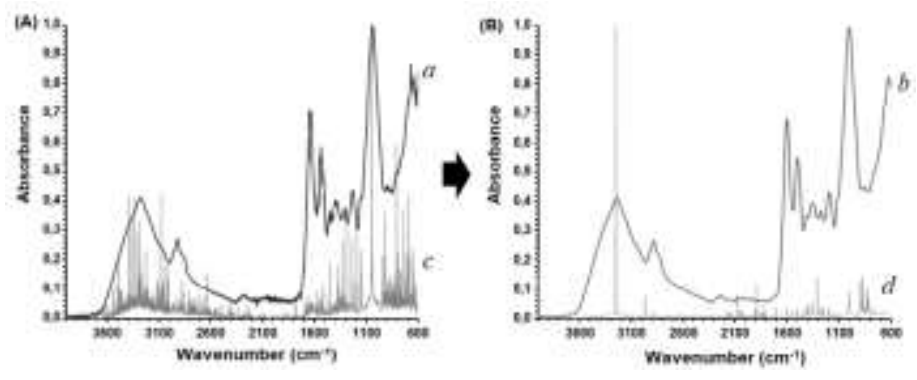

Figure 4. Illustration of effect of the use of FEDS transform without to perform the minimization of residual noise: (A) FEDS transformation without smoothing and (B) FEDS transformation with smoothing using ABSF filter ( $a$ and $b$ are the no-smoothed and smoothed spectra, respectively; whereas $c$ and $d$ denote the FEDS transform in the same order). 
But also, after applying the ABSF algorithm, an adequate reproducibility of FEDS spectra was obtained in all cases. An illustration of excellent correlation obtained between the replicates of $H$. pylori J99 is shown in Figure 5A (values of Pearson correlation coefficients were on average $0.998 \pm 0.005$ ). However, though reproducibility is adequate, the obtaining of corresponding mean spectrum in order to obtain conclusions with greater statistical significance is recommended. Also, it is observed that some signals are strongly decreased in intensity hindering the direct observation. Since FEDS intensity is not directly related with absorbance or with concentration, a high FEDS intensity should not be interpreted as a greater or lesser number of functional groups on the surface. In addition, it is evidenced that a correct application of FEDS should produce a FEDS spectrum with one or more coincident signals, in consequence, it is suggested that, as first step during analysis of FEDS spectrum, direct comparison with its corresponding IR spectrum should be performed (see Figures 5B and 5C). Finally, in Figure 5D an expansion of region among 1600 and $600 \mathrm{~cm}^{-1}$ is shown. Note that, with $N=20$, FEDS spectra, between 1600 and $600 \mathrm{~cm}^{-1}, 25$ signals are identified whereas from IR spectrum 9 signals strongly overlapped are observed.
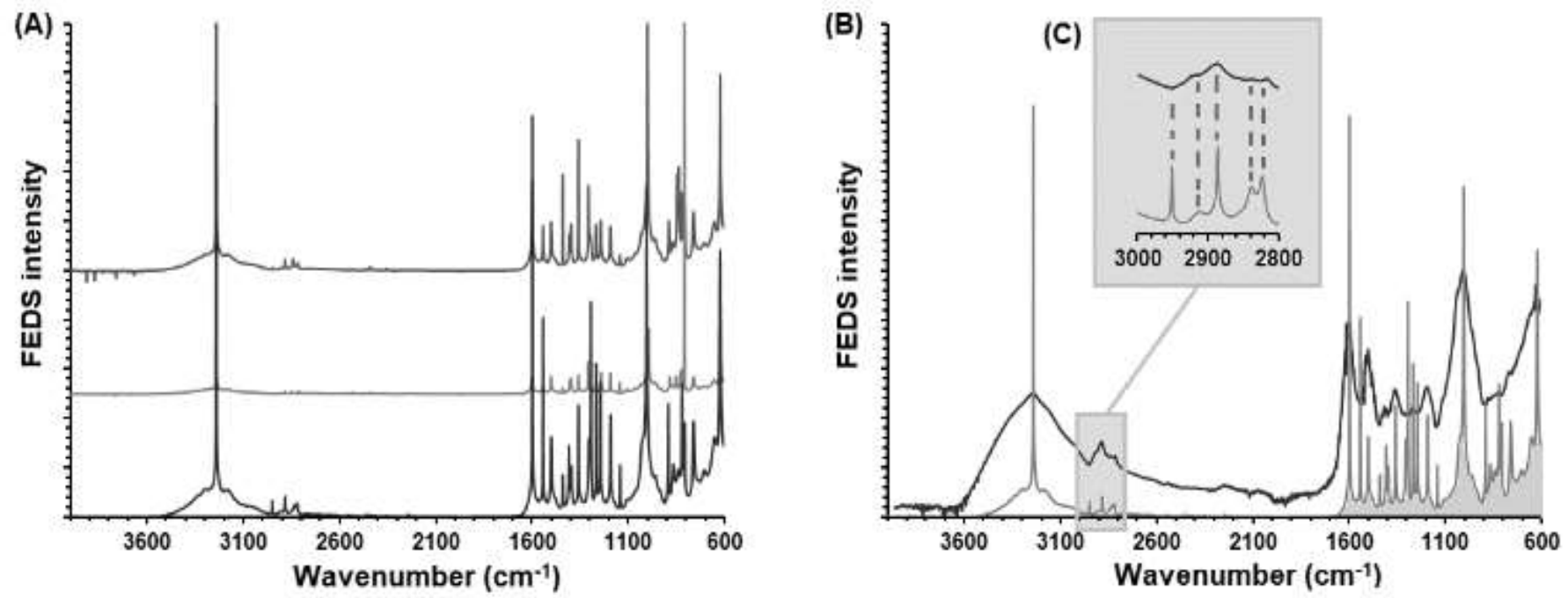

(D)

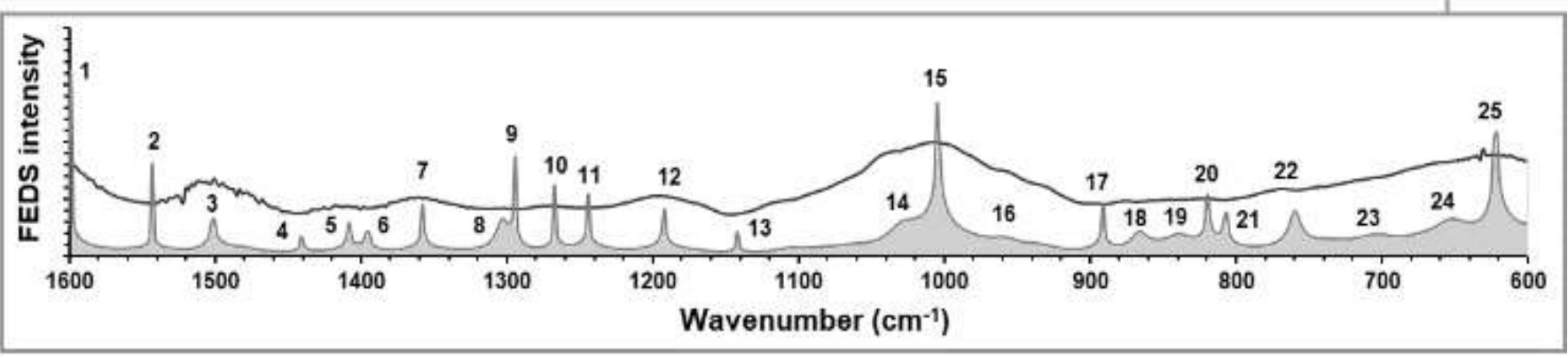

Figure 5. (A) Illustration of reproducibility of FEDS transform applied to mid-IR spectra of H. pylori J99 after to application of ABSF algorithm with $N=20$, (B) comparison of FEDS transform of averaged spectrum of H. pylori J99 and its respective averaged IR spectrum, (C) correlation of FEDS and IR signals in the range from 3000 to $2800 \mathrm{~cm}^{-1}$ and (D) identification of signals obtained by FEDS transform in the range from 1600 to $600 \mathrm{~cm}^{-1}$ compared with the respective mid-IR spectrum.

\subsection{Generalized description of signals}

An important aspect determining the effectiveness of analysis is the correct differentiation of signals. Therefore, these have been classified in two main types: (i) 'true' signals associated with relative maximum values (signals-d) and (ii) 'apparent' signals associated with relative minimum values (signals-k). Here, it is suggested the use of $\mathbf{d}$ and $\mathbf{k}$ in order to avoid confusions, however, there is not no formal reason for this designation. Note that, between 1600 and $600 \mathrm{~cm}^{-1}$, in this specific case, 25 signals were identified, where $d+k=25$ being $d$ and $k$ the number of $\mathbf{d}$ and $\mathbf{k}$ signals, respectively. By direct observation of spectra, this question can be easily resolved (see Figure 6). Thus, it can be concluded that values for this example are $d=17$ and $k=8$.

An important aspect related with the signal-d and signal-k is that a better correlation of different spectra could be performed because the spectral comparison is based on two types of signals. In addition, displacements can be analyzed with respect to signals-k and not only with respect to wavenumber associated with each signal being an alternative to ease the spectral analysis. Another characteristic aspect of signal-k is that these signals are overlapping signals, and therefore, when two signals are located on each side of the same signal-k, it is an evidence that adjacent signals are true signals.

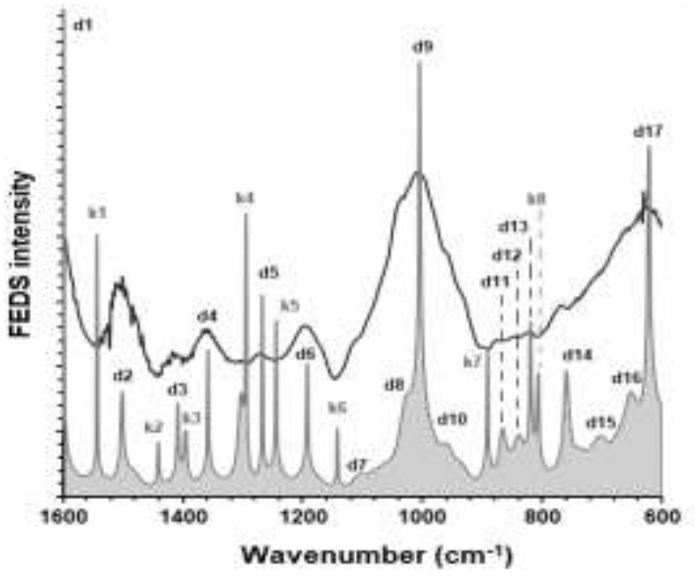

Figure 6. Assignation of signals $\mathbf{d}$ and $\mathbf{k}$ for $H$. pylori 43504 spectrum (pointed red signals correspond to the FEDS spectrum whereas blue line corresponds the experimental spectrum). 


\subsection{Analysis of IR spectra of $H$. pylori biofilms}

Main signals identified in the IR spectra of $H$. pylori are shown in Figure 7. In order to ease the analysis FEDS of spectra, different analysis windows or spectral ranges, were defined: (A) $4000-3000 \mathrm{~cm}^{-1}$, (B) $3000-2500 \mathrm{~cm}^{-1}$, (C) $2500-2000$ $\mathrm{cm}^{-1}$, (D) $2000-1300 \mathrm{~cm}^{-1}$ and (E) $1300-600 \mathrm{~cm}^{-1}$. In addition, a summary of the main signals and their chemical nature is shown in Table 1.

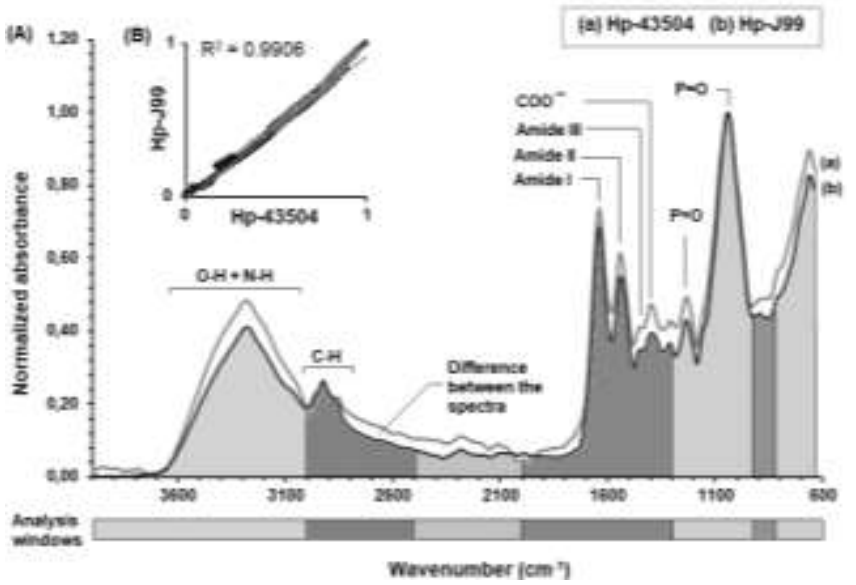

Figure 7. Averaged mid-IR spectra of $H$. pylori strains obtained by ATR technique. (a) H. pylori 43504 and (b) H. pylori J99. The different analysis windows defined for the FEDS application are illustrated by color ranges.

Table 1. Assignation of main signals observed in the average mid-IR spectrum of $H$. pylori biofilms: ATCC J99 and ATCC 43504

\begin{tabular}{|c|c|c|}
\hline $\begin{array}{l}\text { Analysis range } \\
\qquad\left(\mathrm{cm}^{-1}\right)\end{array}$ & $\begin{array}{l}\text { Wavenumber } \\
\left(\mathrm{cm}^{-1}\right)\end{array}$ & Description \\
\hline $3000-4000$ & No specified & $\begin{array}{l}\text { Vibrations associated with } \mathrm{O}-\mathrm{H} \text { and } \mathrm{N}-\mathrm{H} \text { groups. } \\
\text { It is not possible to indicate a specific signal due } \\
\text { to the overlap. This band is affected by the } \\
\text { presence of water, alcohols, amines, etc. }\end{array}$ \\
\hline $2500-3000$ & $2800-3000$ & $\begin{array}{l}\text { Vibrations associated with } \mathrm{C}-\mathrm{H} \text {. These } \\
\text { vibrations can be } \mathrm{C}-\mathrm{H} \text { of methyl groups, } \\
\text { methylene groups or } \mathrm{C}-\mathrm{H} \text { in a ring or cyclic } \\
\text { structure. }\end{array}$ \\
\hline $2000-2500$ & $2240-2350$ & $\begin{array}{l}\text { Vibration of NCO. This signal is not associated } \\
\text { with the bacterial biofilm but that is associated } \\
\text { with spectral marker onto support surface. }\end{array}$ \\
\hline \multirow[t]{4}{*}{$1300-2000$} & 1650 & $\begin{array}{l}\text { Amide I corresponding to } \mathrm{C}=\mathrm{O} \text { stretching } \\
\text { vibration with minor contributions from out-of- } \\
\text { phase } \mathrm{CN} \text { stretching vibration, the } \mathrm{CCN} \\
\text { deformation and the NH in-plane bending. }\end{array}$ \\
\hline & 1550 & $\begin{array}{l}\text { Amide II corresponding to out-of-plane } \\
\text { combination of the } \mathrm{NH} \text { in plane bending and the } \\
\mathrm{CN} \text { stretching vibration with smaller } \\
\text { contributions from the } \mathrm{CO} \text { in plane bending and } \\
\text { the } \mathrm{CC} \text { and } \mathrm{NC} \text { stretching vibrations. }\end{array}$ \\
\hline & 1450 & $\begin{array}{l}\text { Amide III corresponding to the in-phase } \\
\text { combination of NH bending and the } \mathrm{CN} \\
\text { stretching vibration with small contributions } \\
\text { from the } \mathrm{CO} \text { in plane bending and the C-C } \\
\text { stretching vibration. }\end{array}$ \\
\hline & 1400 & $\begin{array}{l}\text { Symmetric stretching of carboxylate group } \\
\text { observed in several bacteria. }\end{array}$ \\
\hline \multirow[t]{2}{*}{$600-1300$} & 1240 & $\begin{array}{l}\text { Vibration of } \mathrm{P}=\mathrm{O}(y 1) \text {. The assignation was } \\
\text { based on models for DNA phosphodiester group } \\
\text { and by comparison with results of other } \\
\text { researchers with different bacteria. }\end{array}$ \\
\hline & 1050 & $\begin{array}{l}\mathrm{P}=\mathrm{O}(y 2) \text {. The assignation was based on models } \\
\text { for DNA phosphodiester group and by } \\
\text { comparison with results of other researchers } \\
\text { with different bacteria. }\end{array}$ \\
\hline
\end{tabular}

These signals are similar to those described for diverse microorganisms in several works [20-21, 36-38] and, though important information can be obtained from them, for specific analysis of outer surface of $\mathrm{H}$. pylori are not very useful. In addition, it can be seen that for both strains the IR spectra are very similar, being the Pearson correlation coefficient $\left(\mathrm{R}^{2}\right)$ calculated about 0.9906 , in consequence, the differentiating information contained in the spectra is lower than $10 \%$. By a classic analysis of IR spectra could be concluded that the two strains under study are the same. In consequence, this first analysis permits to establish that it is not possible to differentiate the target strains of $H$. pylori using the classic method used for the interpretation of IR spectra. However, it is clear that some signals can be used for the monitoring of the surface changes since these are easy and correctly identified.

\subsection{FEDS analysis of $H$. pylori biofilms}

In the region from $4000 \mathrm{~cm}^{-1}$ to $1300 \mathrm{~cm}^{-1}, 39$ signals-d and 13 signals-k were observed for both bacteria (see Figures 8 and 9). In the region from 4000 to 3000 $\mathrm{cm}^{-1}$ is observed the signal associated with $\mathrm{N}-\mathrm{H}$ vibrations, which is explained by the peptides, proteins or aminoglycosides on the surface of the bacterium.

Two signals-d are identified as $\mathbf{d} \mathbf{1}$ and $\mathbf{d} \mathbf{2}$ which are associated with similar vibrations of $\mathrm{N}-\mathrm{H}$ or $\mathrm{O}-\mathrm{H}$ but with a weaker intensity. The first strategy of analysis is to check the presence of water since it is part of living cell and the dried procedure not necessarily warrants the total elimination of water; for the above, previously-published IR and FEDS spectra of water were used [24, 39, 40]; in consequence, by comparison with them, it is suggested that $\mathbf{d 1}$ could be associated with water molecules remains in the external layer.

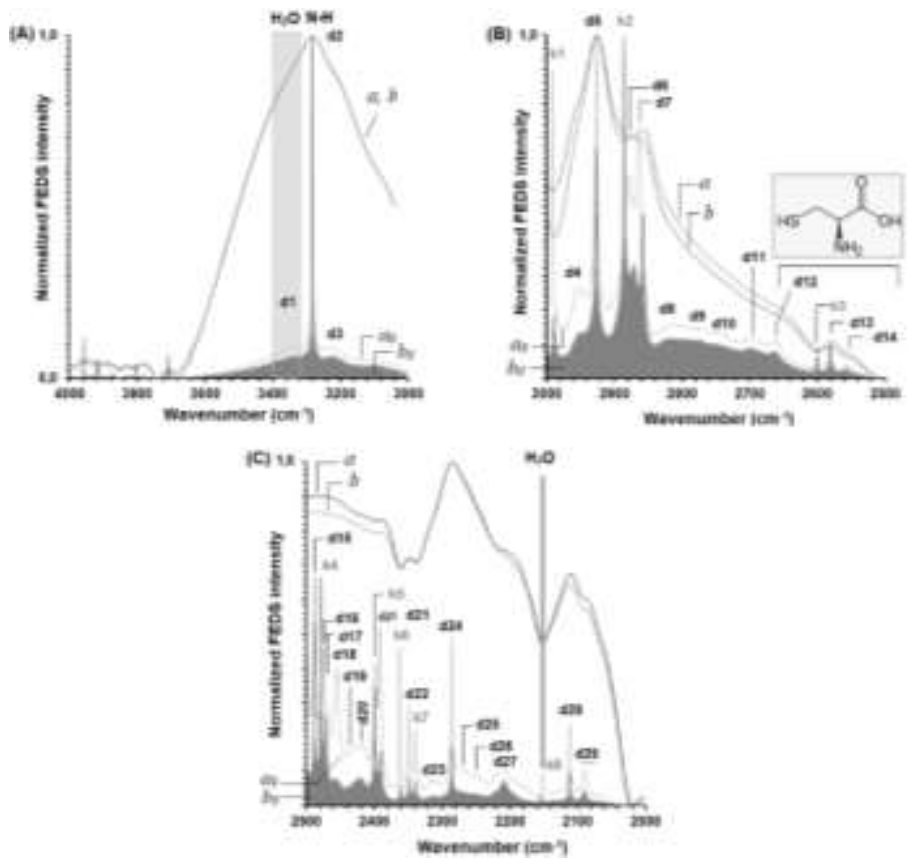

Figure 8. FEDS spectra for averaged mid-IR spectra of H. pylori 43504 ( $a$ and $a_{0}$, respectively) and $H$. pylori $J 99$ ( $b$ and $b_{0}$, respectively): (A) $4000-3000 \mathrm{~cm}^{-1}$, (B) $3000-2500 \mathrm{~cm}^{-1}$ and (C) $2500-2000 \mathrm{~cm}^{-1}$.

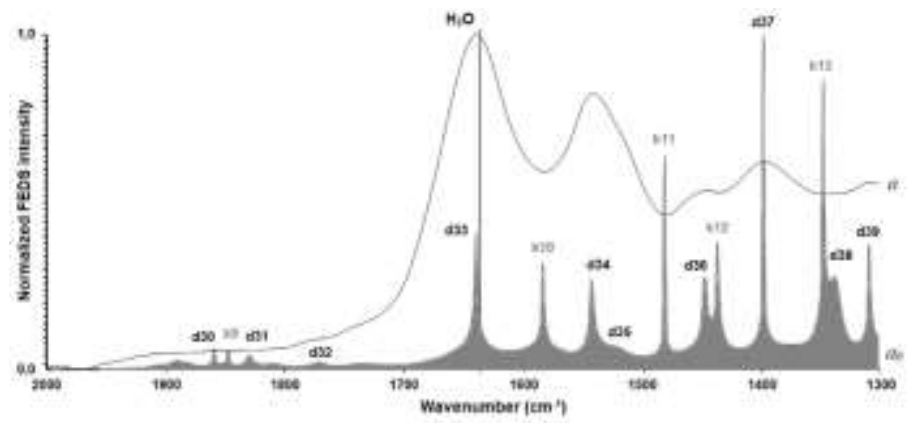

Figure 9. FEDS spectra for averaged mid-IR spectra of H. pylori 43504 and H. pylori J99 (IR spectra, $a$, and FEDS spectra $\left(a_{0}\right)$ were completely overlapped). Window analysis was: $2000-1300 \mathrm{~cm}^{-1}$. 
In order to verify the previous hypothesis, in the spectrum should exist more information related with the presence of water molecules. Thus, for water molecule in liquid phase, in the respective FEDS spectrum, scissor bending at $\sim 1645 \mathrm{~cm}^{-1}$ and a combination signal at $2150 \mathrm{~cm}^{-1}$ has been described [24]; however, it can be seen that, in both spectra, a weak signal at $2150 \mathrm{~cm}^{-1}$ is identified to be $\mathbf{k 8}$, and therefore, since it is defined to be an overlap signal, the presence of this signal cannot be unequivocally associated with water molecules. On the other hand, signal at $1650 \mathrm{~cm}^{-1}$ was evidenced to be equal to $\mathbf{d 3 3}$, in consequence, signals d1 and d3 were assigned to be $\mathrm{OH}$ of alcohols (e.g., glycosides contained in the surface); the above is coherent with the signals FEDS described for low-molecular weight alcohols [24], and with vibrations of $\mathrm{O}-\mathrm{H}$ groups described for pentoses (ribose, 2-deoxy-D-ribose, arabinose and xylose) [41], for N-acetyl-D-glucosamine [42], and for D-glucuronic acid [42], which are components usually identified in Gram negative bacterium biofilms [41-44]. However, presence of adsorbed water molecules is suggested. A summary of main signals is shown in Table 2 .

Table 2. Main signals associate for water [24, 39, 40].

\begin{tabular}{|l|l|l|}
\hline \multicolumn{1}{|c|}{ Technique } & \multicolumn{1}{|c|}{$\begin{array}{c}\text { Wavenumber } \\
\left(\mathbf{c m}^{-1}\right)\end{array}$} & \multicolumn{1}{c|}{ Description } \\
\hline $\begin{array}{l}\text { IR- } \\
\text { spectroscopy }\end{array}$ & $3700-3800$ & $\begin{array}{l}\text { Multiples signals associated with } \mathrm{H}_{2} \mathrm{O}(\mathrm{g}) \\
\text { and } \mathrm{CO}_{2}(\mathrm{~g})\end{array}$ \\
\hline & $3600-3700$ & Small amount of $\mathrm{H}_{2} \mathrm{O}(\mathrm{l})$ \\
\hline & $2300-2350$ & $\begin{array}{l}\text { Multiples small signals associated with } \\
\mathrm{H}_{2} \mathrm{O}(\mathrm{g}) \text { and } \mathrm{CO}_{2}(\mathrm{~g})\end{array}$ \\
\hline & $1620-1550$ & $\begin{array}{l}\text { Multiples small signals associated with } \\
\mathrm{H}_{2} \mathrm{O}(\mathrm{g}) \text { and } \mathrm{CO}_{2}(\mathrm{~g})\end{array}$ \\
\hline & 650 & $\begin{array}{l}\text { Small signal associated with } \mathrm{H}_{2} \mathrm{O}(\mathrm{g}) \text { and } \\
\mathrm{CO}\end{array}$ \\
\hline & 3060 & Asymmetric stretching of $\mathrm{H}-\mathrm{O}-\mathrm{H}$ \\
\hline & 3020 & Symmetric stretching of $\mathrm{H}-\mathrm{O}-\mathrm{H}$ \\
\hline & 2150 & Combination signal (weak intensity) \\
\hline & 1645 & Scissor bending \\
\hline & 1200 & $\begin{array}{l}\text { No identified and no verified by other } \\
\text { sources }\end{array}$ \\
\hline & 700 & No identified \\
\hline
\end{tabular}

Signals in the region between 3000 and $2500 \mathrm{~cm}^{-1}$ are associated mainly with $\mathrm{CH}_{2}$ and $\mathrm{CH}_{3}$ vibrations (from $\mathbf{d} 4$ to $\mathbf{d 7}$ ), since these signals are common for many organic compounds these were considered as not important. However, in the IR spectrum, S-H vibrations are difficultly identified, usually one single signal with weak intensity is described [45-47]. By using of method of linear polarized vibrational spectroscopy, both IR and Raman spectroscopy, the signal at $\sim 2551 \mathrm{~cm}^{-1}$ has been associated to stretching vibration of the SH-group [48], being consistent with signal d14 (Figure 8B). Similar results have been observed during experiments of click chemistry of resins [49]. In particular, by the analysis based on aspect ratio $(q)$, i.e., ratio of the signal height to its width, from results published can be seen that SH signal is a wide signal is observed $\left(q_{\mathrm{SH}}=0.68\right.$ whereas $q_{\mathrm{C}=\mathrm{O}}=4.26$ ) [49], suggesting that some overlapping grade could be present. In amino acids, and in particular, for cysteine, three signals associated with the S-H group are expected from computational analysis by Density Functional Theory or DFT: $\sim 2560, \sim 2580$ and $\sim 2600 \mathrm{~cm}^{-1}$ [45].

These signals were identified for $H$. pylori 43504 and $H$. pylori J99 at 2550 $\mathrm{cm}^{-1}(\mathbf{d 1 3}), 2580 \mathrm{~cm}^{-1}(\mathbf{d 1 4})$ and $2600 \mathrm{~cm}^{-1}(\mathbf{d 1 5})$. In addition, for cysteine, a signal associated with N-H vibrations is also identified at $2660 \mathrm{~cm}^{-1}$ corresponding to $\mathbf{d 1 2}$ [45]; the importance of this signal is that it can be used as a marking signal to facilitate the location of $\mathrm{S}-\mathrm{H}$ signals during the analysis of spectra. However, it is important to look for more characteristic signals of this amino acid in order to evaluate the coherence in the analysis. Thus, S-H in-plane bending is expected at $1064 \mathrm{~cm}^{-1}$ and C-S stretching at $692 \mathrm{~cm}^{-1}$ [45]. Both signals were identified and named as $\mathbf{d 4 3}$ and $\mathbf{d 5 2}$, respectively (see Figure 10).

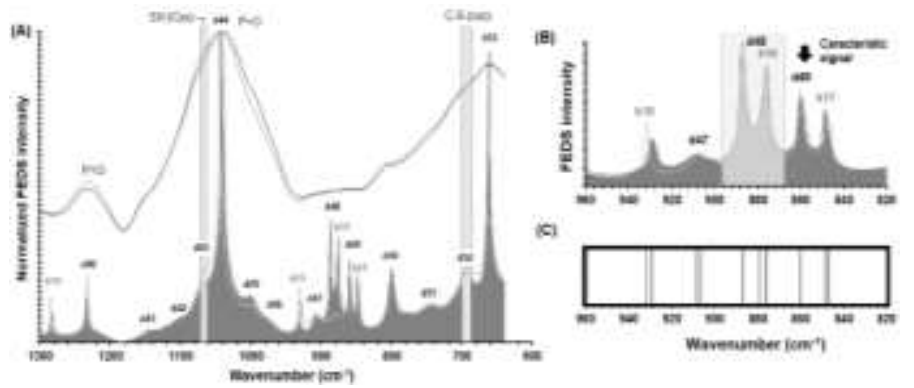

Figure 10. FEDS spectra for averaged mid-IR spectra of $H$. pylori 43504 (Dotted red and black lines) and H. pylori J99 (green and black lines, respectively) between 1300 and $600 \mathrm{~cm}^{-1}$ (A) and 960 and $820 \mathrm{~cm}^{-1}$ (B). Comparison of FEDS spectra for bacteria by discontinuous pattern of signals (C).

The above is coherent with the analysis of outer membrane protein families where have been identified cysteine residues [50], in particular, they worked, among other, with the strain $\mathrm{H}$. pylori $\mathrm{J} 99$ that is the same strains used by us. In addition, the protein designed HpcA (H. pylori cysteine-rich protein $\mathrm{A}$ ) have been identified to be produced by $H$. pylory gene HP0211, and it is characterized by a molecular weight of $27.3 \mathrm{kDa}$ containing 250 amino acids, of which 14 (5.6 $\%)$ is cysteine $[51,42]$. Other outer membrane proteins characterized by high content of cysteine are $\mathrm{HpcC}$ (gene HP1098) and cysteine-rich protein X (HP1117) among others [53, 54].

In the range between 2500 and $2000 \mathrm{~cm}^{-1}$ the signal associated with the spectral marker (signal $\alpha$ ) is expected (see Table 3 ). Spectral marker used has units of phenyl isocyanate on its structure, therefore, the analysis was directed toward the identification of signals associated with the group -NCO; thus, signals identified were the vibration of $\mathrm{N}=\mathrm{C}=\mathrm{O}$ at $2285 \mathrm{~cm}^{-1}(\mathbf{d 2 4})$ and $2265 \mathrm{~cm}^{-1}(\mathbf{d 2 5})$. These signals have been described in the range of $2280-2230 \mathrm{~cm}^{-1}$ [55], and exactly at $2285 \mathrm{~cm}^{-1}$ [56]. One additional vibration of $\mathrm{NCO}$ was identified at $2210 \mathrm{~cm}^{-1}$ (d27) which have been described in the range $2260-2130 \mathrm{~cm}^{-1}$ [55]. Other four no-fundamental signals are associated with the marker and were satisfactorily identified: $2345 \mathrm{~cm}^{-1}$ (d22), $2390 \mathrm{~cm}^{-1}$ (d21), $2700 \mathrm{~cm}^{-1}$ (d11) and $2870 \mathrm{~cm}^{-1}$ (d6) which have been previously described at $2340,2370,2700$ and $2870 \mathrm{~cm}^{-1}$ [56].

Signals associated with $\mathrm{PO}_{2}{ }^{-}$groups have been described from the study of ribose-phosphate links at $1230-1244 \mathrm{~cm}^{-1}$ and $1084-1089 \mathrm{~cm}^{-1}$ [57], however, according to the first IR spectrum analysis, these signals were assigned at 1240 $\mathrm{cm}^{-1}(\mathbf{d 4 0})$ and $1050 \mathrm{~cm}^{-1}(\mathbf{d 4 4})$ which were previously identified in the IRspectrum $(\mathrm{P}=\mathrm{O})$. However, the assignment of signal $\mathbf{d 4 4}$ should be revised. Note that, from analysis computational of dimethyl phosphate, four signals have been described: $1205 \mathrm{~cm}^{-1}$ ( $\mathrm{P}=\mathrm{O}$ asymmetric stretching), $1083 \mathrm{~cm}^{-1}$ ( $\mathrm{P}=\mathrm{O}$ symmetric stretching), $824 \mathrm{~cm}^{-1}$ (P-O asymmetric bending) and $760 \mathrm{~cm}^{-1}$ (P-O symmetric bending), in addition, the same four signals have been obtained for ethyl methyl phosphate but with significant displacements [58]. The above suggests that it should not be expected to obtain the same positions since the nature of groups linked to phosphate units are very different. In consequence, the results published by Heidari (2016) are expected to be a better comparison with the systems under study. Other researchers have described four signals with positions very similar to those described by Heidari, these are $1241 \mathrm{~cm}^{-1}\left(\mathrm{PO}_{2}^{-}\right), 1110 \mathrm{~cm}^{-1}\left(\mathrm{PO}_{2}^{-}\right), 801$ $\mathrm{cm}^{-1}$ (OPO) and $751 \mathrm{~cm}^{-1}$ (OPO) [36]. In addition, Hidari (2016) also describes two signals at $970 \mathrm{~cm}^{-1}$ and $916 \mathrm{~cm}^{-1}$. In consequence, signals assigned were 1235 $\mathrm{cm}^{-1}(\mathbf{d 4 0}), 1045 \mathrm{~cm}^{-1}(\mathbf{d 4 4})$ (taking in consideration that other signals possibly can be associated with $\mathbf{d 4 2}$, these are: $970 \mathrm{~cm}^{-1}(\mathbf{d 4 6}), 916 \mathrm{~cm}^{-1}(\mathbf{d 4 7}), 801 \mathrm{~cm}^{-1}$ (d50) and $745 \mathrm{~cm}^{-1}(\mathbf{d 5 1})$.

Signals associated with amine I, amine II, amine III and COO were identified by direct comparison of IR spectra: $1640 \mathrm{~cm}^{-1}(\mathbf{d 3 3}), 1540 \mathrm{~cm}^{-1}(\mathbf{d 3 4}), 1445 \mathrm{~cm}$ ${ }^{1}$ (d36) and $1400 \mathrm{~cm}^{-1}$ (d37). Finally, it can be seen that a sub-range defined between $820 \mathrm{~cm}^{-1}$ and $960 \mathrm{~cm}^{-1}$ appears as a characteristic pattern in the spectra. The above is based on: (i) signal $\mathbf{d 4 9}$ is completely the same in both strains, but at the same time, signals $\mathbf{~} 4 \mathbf{4 7}, \mathbf{k 1 5}$ and $\mathbf{k 1 7}$ appear to be slightly displaced and (ii) two signals in the spectrum of $H$. pylori $\mathrm{J} 99$ (d48 and k16) are not identified in the spectrum of $H$. pylori 43504 which shows only one signal (see figure 10B and 10C). The above suggests that it is possible to define a comparison pattern in order to discriminate between these microorganisms, however, the above hypothesis should be experimentally evaluated. 
Table 3. Main signals associate with group isocyanate on the phenyl isocyanate $[55,56]$.

\begin{tabular}{|c|c|c|}
\hline Technique & Wavenumber $\left(\mathrm{cm}^{-1}\right)$ & Description \\
\hline \multirow[t]{5}{*}{ IR- spectroscopy } & 2285 & Asymmetric stretching of -NCO \\
\hline & 2210 & Stretching of $\mathrm{OC} \equiv \mathrm{N}$ \\
\hline & 1448 & $-\mathrm{NCO}$ \\
\hline & 632 & $-\mathrm{NCO}$ \\
\hline & 589 & $-\mathrm{NCO}$ \\
\hline \multirow[t]{8}{*}{ FEDS $^{(1)}$} & 2870 & No-fundamental vibration \\
\hline & 2700 & No-fundamental vibration \\
\hline & 2390 & No-fundamental vibration \\
\hline & 2345 & No-fundamental vibration \\
\hline & 2285 & $\begin{array}{l}\text { Fundamental vibration of -NCO } \\
\text { (Asymmetric stretching) }\end{array}$ \\
\hline & 2265 & $\begin{array}{l}\text { Fundamental vibration of -NCO } \\
\text { (Asymmetric stretching) }\end{array}$ \\
\hline & 2210 & Stretching of $\mathrm{OC} \equiv \mathrm{N}$ \\
\hline & 1448 & Fundamental vibration of -NCO \\
\hline
\end{tabular}

(1) FEDS signals described in the table were determined from experimental results and compared satisfactorily with previous publications

\section{CONCLUSIONS}

FEDS transform of IR spectrum is a powerful analytical tool for the improvement of analysis of microorganism IR spectra. For correct application of technique, a minimum amount of noise in the working spectra is required; in addition, reproducibility should be warrant by the implementation of standardized protocols and the use of an appropriate number of samples.

On the other hand, results obtained by FEDS demonstrate the capacity of FEDS for the improvement of analysis of IR spectra of microorganisms. In the particular case of $H$. pylori strains, it was observed that, by FEDS, is possible the deconvolution of signals, permitting to obtain a better differentiation of them. In this way, it was evidenced that vibrational spectrum of $H$. pylori can be understood considering the main components of outer membrane cell, and therefore, it is concluded that good results in the spectral characterization of $H$. pylori surface can be obtained from IR spectra of individual bacterial components

\section{ACKNOWLEDGMENTS}

Sixta Palencia thanks to Conicyt-Chile, Universidad de Concepción (Chile) and Universidad del Valle (Colombia) by the funds for the performing of the project and doctoral scholarship. Authors thanks to Mindtech s.a.s. by the support into acquisition of spectral marker.

\section{CONFLICTS OF INTEREST}

The authors declare that they have no conflict of interest.

\section{REFERENCES}

1. S. Phandis, M. Parlow, M. Levy, D. Ilver, C. Caulkins, J. Connors, B. Dunn, Infect. Immun., 64, 905 (1996).

2. M. Chmiela, E. Czkwianianc, T. Wadstrom, W. Rudnicka, Gut 40, (1997).

3. A.M. Alkout, C.C. Blackwell, D.M. Weir, I.R. Poxton, R.A. Elton, W. Luman, K. Palmer, Gastroenterol., 112, 1179 (1997).

4. G. Sachs, D. Scott, D. Weeks, K. Melchers, Trends Microbiol., 9, 532 (2001).

5. S.L. Palencia, G. Lagos, A. García, J. Sci. Technol. Appl., 1, 53 (2016)
6. V. Marqués, B. Cunha, A. Couto, P. Sampaio, LP. Fonseca, S. Aleixo, CRC. Calado, Spectrochim. Acta A: Mol. Biomol. Spectr., 210, 193 (2018).

7. H. Zhao, Y. Wu, Z. Xu, R. Ma, Y. Ding, X. Bai, Q. Rong, Y. Zhanng, B. Li, X. Ji, Front. Microbiol., 10, 1 (2019).

8. G. Bode, F. Mauch, H. Ditschuneit, P. Malfertheiner, J. General Microbiol., 139, 3029 (1993).

9. B.E. Dunn, N.B. Vakil, B.G. Schneider, M.M. Miller, J.B. Zitzer, T. Peutz. Infection and Immunology., 65, 1181 (1997).

10. M.R. Amievalow, E.M. El-Omar, Gastroenterol., 134, 306 (2008).

11. H. Shimomura, K. Hosoda, S. Hayashi, K. Yokota, Y. Hirai, J. Bacteriol., 194, 2658 (2012).

12. A. Moran. FEMS Immunol. Medical Microbiol., 10, 271 (1995).

13. N. Sabarth, S. Lamer, U. Zimny-Amdt, P.R. Jungblut, T.F. Meyer, D. Bumann. J. Biol. Chem., 277, 27896 (2002).

14. H.H. Tuson, D.B. Weibel. Soft Matter. 9, 4368 (2013).

15. S. Kumar, A.K. Rai, V.B. Singh, S.B. Rai. Spectrochim. Act Part A: Mol. Biomol. Spectr. 61, 2741 (2005).

16. M. Fischer, G.J. Triggs, T.F. Krauss. Appl Environ. Microbiol. 82, 1362 (2016).

17. A. Elbourne, J. Chapman, A. Gelmi, D. Cozzolino, R.J. Crawford, V.K Truong. J. Colloid Interf. Sci. 546, 192-210 (2019).

18. F. Abbasian, E. Ghafar, S. Magierowsky. Bioengineering (Basel). 5, 1 (2018).

19. A. Alvarez-Ordoñez, D. Mouwen, M. López, M. Prieto, J. Microbiol. Met. 84, 369 (2011).

20. J. Ojeda, M. Dittrich in: Microbial systems biology: Methods and Protocols, Methods in Molecular Biology, Navid A. Springer Science, 2012, pp. $187-$ 211.

21. J. Prakash, S. Kar, C. Lin, C.Y. Chen, C.F. Chang, J.S. Jean, T.R. Kulp. Spectrochim. Act Part A: Mol. Biomol. Spectr. 116, 478-484 (2013).

22. W. Friesen, K. Michaelian, Appl. Spectr. 45, 50-56 (1991).

23. T. Vazhnova, D. Lukyanov. Anal. Chem. 85, 11291-11296 (2013).

24. M. Palencia, J. Adv. Res.14, 53 (2018).

25. P. Kowalski, R. Smyk. 2018 International Interdisciplinary PhD Workshop (IIPhDW), 277 (2018)

26. M. Palencia, V. Garces-Villegas, D.F. Restrepo, J.M. Martínez, L.R. AnayaTatis, E.M. Combatt. J. Appl. BIotechnol. Bioing. 7, 43 (2020).

27. Y. Sánchez, A. García-Quintero, M. Palencia. J. Sci. Technol. Appl. 5, 45 (2018).

28. A. García-Quintero, E. Combatt, M. Palencia. J. Sci. Technol. Appl. 4, 28 (2018).

29. A. Otálora, T.A. Lerma, M. Palencia. J. Sci. Technol. Appl. 7, 5 (2019).

30. T.A. Lerma, V. Garces, M. Palencia. Eur. Polym. J. 128, 109627 (2020).

31. M. Palencia, M. Mora, T. Lerma. Smart Sustain. Built Environ. DOI: 10.1108/SASBE-09-2019-0126 (2020)

32. M. Palencia, T. Lerma, N. Afanasjeva. Eur. Polym. J.115, 212 (2019).

33. L.R. Anaya, K.H. Libreros, V.J. Palencia, V.J. Atencio, M. Palencia. J. Sci. Technol. Appl. 6, 96 (2019).

34. A. Otalora, M. Palencia, J. Sci. Technol. Appl. 6, 96 (2019).

35. S. Palencia, A. García, M. Palencia. Appl. Spectr. Manuscript accepted. Publication date (2021)

36. Y. Guan, C.J. Wurrey, G.J. Thomas. Biophys. J., 66, 225 (1994)

37. Y. Guan, G.J. Thomas. Biopolym. 39, 813 (1996).

38. W. Jiang, A. Saxena, B. Song, B.B. Ward, T.J. Beveridge, S. Myneni. Langmuir. 20, 11433 (2004).

39. E. Pretsch, C. Bühlmann, C. Affolter, C. Herrera, R. Martínez, Determinación estructural de compuestos orgánicos, Barcelona, Ed. Masson., 2005, $481 \mathrm{p}$.

40. F.O. Libnau, O.M. Kvalheim, A.A. Christy, J. Toft. Vibr. Spectr. 7, 243 (1994).

41.E. Wiercigroch, E. Szafraniec, K. Czamara, M.Z. Pacia, K. Majzner, K. Kochan, A. Kaczor, M. Baranska, K. Malek. Spectrochim Acta Part A: Molec Biomol Spectr. 185, 317 (2017)

42. A. Kovacs, B. Nyerges, V. Izvekov. J Phys Chem., 112, 5728 (2008).

43. A. Moran. FEMS Immunol. Medical Microbiol., 10, 271 (1995).

44. A. Moran, In: Helicobacter pylori: Physiology and Genetics. Mobley HLT, Mendz GL, Hazell SL (Eds). ASM Press. Washington DC, USA, 2001.

45. S. Parker. Chem. Phys. 424, 75-79 (2013).

46. Z. Xia, L. Baird, N. Zimmerman, M. Yeager. Appl. Surf. Sci. 416, 565 (2017)

47. V. Lacour, K. Moumanis, W. Hassen, C. Elie-Caille, T. Leblois, J. Dubowski. Langmuir 35, 4415 (2019) 
48. B. Koleva, M. Spiteller, T. Kolev. Amino Acids 38, 295 (2010)

50. C. Dumrese, L. Slomianla, U. Ziegler, S.S. Choi, A. Kalia, A. Fulujira, W. Lu, D. Berg, M. Benghezal, B. Marshall, P. Mittl. FEBS. Lett. 583, 1637 (2009).

51. R.A. Alm, J. Bina, B. Andrews, P. Doig, R. Hanckck, T. Trust, Infect Immun., 68, 4155 (2000).

52. P. Cao, M.S. McClain, M.H. Forsyth, T. Cover. Infect. Immun., 66, 2984 (1998).

53. P.R. Mittl, L. Luthy, P. Hunziker, M. Grutter. The J. Biol. Chem., 275, 17693 (2000).
49. Nagarjuna, M. Saifullah, R. Ganesan. RSC Adv. 8, 11403 (2018)

54. G. Zanottu, L. Cendron. Worl J. Gastroenterol., 20, 1402 (2014).

55. E. Pretsch, C. Bühlmann, C. Affolter, C. Herrera, R. Martínez, "Determinación estructural de compuestos orgánicos", Barcelona, Ed. Masson., 2005.

56. C.V. Stephenson, W.C. Coburn, W.S. Wilcox. Spectrochim. Acta., 17, 933946 (1961).

57. A. Heidari, Austin J. Analyt. Pharm. Chem., 3, 1058 (2016)

58. V. Andrushchenko, L. Benda, O. Pav, M. Dracinsky, P. Bour. The J. Phys. Chem B. 119, 10682 (2015). 